\title{
AS REPRESENTAÇÕES SOCIAIS DE ESTUDANTES DE EDUCAÇÃO FÍSICA SOBRE A FORMAÇÃO DE PROFESSORES
}

\author{
PHYSICAL EDUCATION STUDENTS' SOCIAL REPRESENTATIONS ON \\ TEACHER TRAINING
}

\section{LAS REPRESENTACIONES SOCIALES DE LOS ESTUDIANTES DE EDUCACIÓN FÍSICA SOBRE LA FORMACIÓN DE DOCENTES}

\author{
Felipe da Silva Triani, Carlos Alberto de Oliveira Magalhães Júnior*, \\ Cristina Novikoff***
}

Palavras chave:

Educação Física.

Docentes.

Conhecimento.

\begin{abstract}
Resumo: Objetivou-se identificar e analisar algumas das representações sociais possíveis que os estudantes do curso de Educação Física de uma universidade privada na Baixada Fluminense do Rio de Janeiro podem apresentar sobre a formação de professores. Assim, 16 estudantes de um curso de Educação Física responderam a um questionário cujas respostas foram avaliadas via análise de conteúdo. Os resultados apontam os professores como uma panaceia para os estudantes e, esses, em maioria, não souberam atribuir sentido à formação de professores, mas há o consenso de que o "conhecimento" é importante, mesmo considerando que mais da metade ingressou no curso por afinidade. Portanto, foi concluído que um dos principais indícios das representações sociais que estão presentes no senso comum do grupo de estudantes é que o conhecimento é o elemento mais expressivo na formação de professores e ainda que possuí-lo é a característica mais marcante do bom professor de Educação Física.
\end{abstract}

\section{Keywords:}

Physical Education.

Faculty.

Knowledge.

Palabras clave: Educación Física. Docentes. Conocimiento.
Abstract: This study aimed to find and analyze some of the possible social representations about teacher training held by Physical Education students at a private university in Rio de Janeiro's Baixada Fluminense area. Sixteen students from a Physical Education school answered a questionnaire and their answers were evaluated through content analysis. The results say students see professors as a panacea and most of them were not able to ascribe meaning to teacher training, but there was a consensus that "knowledge" is important, even though most students entered the course for affinity. Therefore, the study concluded that one of the main indications of social representations present in the student group's common sense is that knowledge is the most expressive element in teacher training and that having it is the most striking characteristic of the good Physical Education teacher.

Resumen: El objetivo de este estudio fue identificar y analizar algunas de las posibles representaciones sociales que los alumnos del curso de Educación Física de una universidad privada en el estado de Río de Janeiro pueden presentar sobre la formación de profesores. Dieciséis estudiantes del curso de Educación Física respondieron a un cuestionario y las respuestas fueron sometidas a análisis de contenido. Los resultados indican que maestros son una panacea para los estudiantes. En su mayoría, no supieron cómo dar sentido a la formación del profesorado, pero existe consenso en cuanto a que el "conocimiento" es importante. Se concluyó que uno de los principales indicios de las representaciones sociales que está presente en el sentido común del grupo de estudiantes es que el conocimiento es el elemento más expresivo en la formación de los profesores y que poseerlo es la característica más llamativa del buen maestro de Educación Física.
*Faculdade Gama e Souza. Rio de Janeiro, RJ, Brasil.

E-mail: felipetriani@gmail.com

**Universidade Estadual de Maringá. Maringá, PR, Brasil.

E-mail: juniormagalhaes@ hotmail.com

***Universidade Federal de Campina Grande. Campina Grande, PB, Brasil. E-mail: cristinanovikoff@gmail.com

Recebido em: 18-10-2016 Aprovado em: 10-04-2017

(c) (1) (8) Licence 


\section{INTRODUÇÃOO}

A Teoria das Representações Sociais apresentada por Moscovici (1978; 2012), atualmente, vem mostrando seu valor num duplo papel da pesquisa científica. 0 primeiro referese à necessidade de se compreender a realidade em que se pretende realizar a pesquisa, já o segundo consiste em intervir na realidade que se propõe a compreender. Neste contexto, concorda-se com Gibbons e seus colaboradores (1994), que afirmam a necessidade da ciência trabalhar em um modo de construção do conhecimento dentro de um contexto de aplicabilidade.

Para Jodelet (2001, p.22), a Representação Social (RS) é uma "[...] forma de conhecimento, socialmente elaborada e compartilhada, com um objetivo prático, e que contribui para a construção de uma realidade comum a um conjunto social", bem como os valores desses estudantes que norteiam tais representações sociais.

A representação social como mecanismo de produção de conhecimento tem a função de tornar conhecido o que era desconhecido, nas palavras de Moscovici (1978; 2012), tornar familiar o que outrora era não familiar. Neste sentido, existem dois processos que tornam possível esse mecanismo, que 0 autor intitula de ancoragem e objetivação.

Ancoragem é o processo em que o indivíduo se depara com um objeto estranho e tenta torná-lo conhecido a partir das imagens já construídas intrinsecamente. Em Horochovski (2004, p. 98), "[...] ancorar é classificar, nomear, rotular e, obviamente, representar". Este contexto de rotular o que não tinha rótulo e/ou nomear o que não tinha nome é o processo de tornar familiar o que era não familiar.

Objetivação é o processo em que o indivíduo descobre o conceito do objeto e o compara a algo real. Esse processo acontece em dois momentos, no primeiro o conceito do objeto é relacionado com sua imagem, logo é descoberto o nome do objeto; já no segundo momento, após compreender o conceito do objeto, ele é comparado com algo tangível e isso é "[...] encher o que está naturalmente vazio, com substância" (MOSCOVICI, 1978, p. 71), ou seja, dar sentido ao objeto, tornando familiar o que era não familiar.

Estudos recentes têm apontado uma necessidade de investimento científico na formação de professores (TARDIF; LESSARD, 2005; MACHADO, 2008; NEGRI et al., 2015). $\mathrm{Na}$ tentativa de identificar a falta de investigação na Educação Física sobre esse objeto de estudo, alguns estudos foram alcançados, a saber: um deles foi realizado no contexto da Educação Física na escola e analisou a representação social que os educandos tinham sobre a disciplina (VEIRA, 2006); outro faz referência à representação social apresentada pelo corpo docente da Educação Infantil sobre a Educação Física (MELLO et al., 2012); e ainda um que discute a representação social de professores de Educação Física sobre a formação continuada (LOUREIRO; CAPARROZ; BRACHT, 2015)

Diante desse cenário epistemológico, percebe-se uma lacuna no que tange à representação social sobre a formação de professores de Educação Física, no contexto da formação inicial. De acordo com Mazzotti (1997) e Wagner (1998), as representações sociais orientam os comportamentos e as práticas sociais. Num contexto educacional, identificar as representações sociais possibilita compreender 0 comportamento e como 0 grupo social interage com 0 objeto. Dessa maneira, torna-se justificável desenvolver uma pesquisa com estudantes de Educação Física ainda na graduação sobre a formação de professores. 
Essa necessidade também se apresenta considerando a timidez nos estudos que se apropriam da Teoria das Representações Sociais na/para produção da ciência. Recentemente foi desenvolvido um estudo no banco de dados da Coordenação de Aperfeiçoamento de Pessoal de Nível Superior (Capes) em que se disponibilizam resumos de teses e dissertações da pós-graduação brasileira. Nele, encontrou-se uma baixa quantidade na ocorrência de estudos em representações sociais no contexto da Educação Física (TRIANI, NOVIKOFF, 2013), entretanto, segundo o estudo, dos trabalhos que foram encontrados nenhum objetivou estudar o fenômeno das representações sociais na formação de professores em Educação Física. Isso implica ressaltar a necessidade de que novos estudos sejam desenvolvidos nesta perspectiva, ainda pouco explorada na Educação Física.

Nesse cenário, pesquisas a partir da iniciação científica vêm sendo desenvolvidas, pensando e discutindo a forma de entender as representações sociais e sua influência na formação inicial de professores. Os resultados poderão ser trabalhados para encontrar caminhos metodológicos que levem os estudantes a uma maior apropriação da ciência. Nesse contexto, o objetivo deste estudo foi identificar e analisar algumas das representações sociais possíveis que os estudantes do curso de Educação Física de uma universidade privada na Baixada Fluminense do Rio de Janeiro podem apresentar sobre a formação de professores.

Sendo assim, a premissa é a de que a Teoria das Representações Sociais de Serge Moscovici (1978) permite compreender como os grupos sociais entendem determinado objeto e daí ter pistas de como melhorar esta compreensão e suas ações. Acredita-se que investigando as representações sociais destes estudantes será possível compreender suas práticas acadêmicas.

\section{METODOLOGIA}

Para atingir o objetivo proposto foi utilizada uma pesquisa de campo com abordagem qualitativa (BAUER; GASKELL, 2003; GÜNTHER, 2006; CRESWELL, 2007), adotando ainda o modelo das Dimensões Novikoff, que prevê cinco fases da pesquisa que se articulam dialeticamente (NOVIKOFF, 2010). A primeira, denominada epistemológica, delineia o estado do conhecimento, o objeto e seus pressupostos a partir da problemática levantada. Na sequência é apresentado o corpus teórico, na dimensão denominada teórica. No encadeamento textual, apresenta-se a dimensão técnica e se faz o detalhamento do procedimento metodológico do estudo. Segue para a apresentação da dimensão morfológica, em que se apresentam os dados coletados no campo e, finalmente, é tecida a dimensão analítico-conclusiva, momento crucial de entrelaçamento entre teoria e dados.

Os sujeitos da pesquisa foram graduandos do curso de Educação Física de uma universidade na Baixada Fluminense no Rio de Janeiro. A escolha foi aleatória e dois alunos de cada período do curso de Licenciatura em Educação Física, totalizando 16 sujeitos, participaram. Não foi realizado nenhum tipo de cálculo amostral porque é uma pesquisa não probabilística e o objetivo não foi compreender a representação do curso, mas do grupo de alunos que participaram.

Utilizamos como instrumento de pesquisa um questionário estruturado previamente testado (CZAJA; JOHNNY, 1996). Esse instrumento consistiu em quinze questões sobre a formação de professores, sendo duas sobre os problemas didático-pedagógicos e político- 
administrativos, quatro sobre a formação docente, legislação e formação continuada, duas sobre características acerca da docência, três sobre as atividades docentes e ainda quatro sobre o contexto de sua formação. Para a análise dos dados foram adotadas as categorias, a priori, propostas por Novikoff (2006), que distribui os tipos de conhecimento em valores socioprofissionais, normativos, gnosiológicos, sociorrelacionais e identitários.

O estudo foi aprovado pelo Comitê de Ética em Pesquisa da Universidade do Grande Rio - UNIGRANRIO, sob guarda do CAAE de número 11200412.2.0000.5283 e realizado com aprovação dos discentes dos cursos de Educação Física envolvidos após assinatura do Termo de Consentimento Livre e Esclarecido (TCLE).

\section{RESULTADOS E DISCUSSÃO}

O questionário, composto de 15 questões abertas e fechadas, foi utilizado para identificar quais as representações sociais que os estudantes têm sobre sua formação. Todavia, serão expostos os resultados desse instrumento por meio de uma análise da categoria de valores proposta por Novikoff (2006).

O Quadro 1 sintetiza as categorias e as questões concernentes a elas:

Quadro 1 - Distribuição das questões frente à categoria de valores

\begin{tabular}{|c|c|c|c|c|c|}
\hline Categorias & $\begin{array}{c}\text { Valores } \\
\text { Socioprofissionais }\end{array}$ & $\begin{array}{c}\text { Valores } \\
\text { Normativos }\end{array}$ & $\begin{array}{c}\text { Valores } \\
\text { Gnosiológicos }\end{array}$ & $\begin{array}{c}\text { Valores } \\
\text { Sociorrelacionais }\end{array}$ & $\begin{array}{c}\text { Valores } \\
\text { Identitários }\end{array}$ \\
\hline $\begin{array}{c}\text { Tipos de } \\
\text { conhecimento }\end{array}$ & $\begin{array}{c}\text { Conhecimento } \\
\text { da dimensão } \\
\text { socioafetiva } \\
\text { do estudante } \\
\text { sobre a } \\
\text { formação } \\
\text { docente }\end{array}$ & $\begin{array}{c}\text { Conhecimento } \\
\text { do estudante } \\
\text { com relação à } \\
\text { caracterização } \\
\text { docente }\end{array}$ & $\begin{array}{c}\text { Conhecimento do } \\
\text { estudante sobre } \\
\text { as atividades } \\
\text { docentes }\end{array}$ & $\begin{array}{c}\text { Conhecimento } \\
\text { sobre 0 } \\
\text { contexto }\end{array}$ \\
\hline Questões & Q11, Q12 & $\begin{array}{c}\text { Q1, Q2, Q3, } \\
\text { Q13 }\end{array}$ & Q4, Q8 & Q6, Q9, Q14 & $\begin{array}{c}\text { Q5, Q7, Q10, } \\
\text { Q15 }\end{array}$ \\
\hline
\end{tabular}

Fonte: Elaborado pelos autores.

Diante do exposto, para melhor compreensão serão apresentadas as categorias separadamente:

\subsection{Valores socioprofissionais}

Ao perguntar aos alunos sobre as dificuldades didático-pedagógicas que existem durante 0 curso, $63 \%$ dos estudantes recorrem aos "professores" para superá-las, $25 \%$ aos "livros" e 19\% utilizam-se de outros meios. Esses dados se assemelham aos estudos de Albuquerque e outros (2009), que apresentam o professor como responsável pela elucidação das dificuldades, pois há representações de ser aquele que detém o conhecimento. No entanto, é possível afirmar com Santos e outros (2012) que muitas das dificuldades não são superadas no curso e somente serão no estágio profissional ou na prática enquanto regente.

Há também dificuldades político-administrativas e, quando isso ocorre, os "professores" são os mais procurados para resolvê-las, com $63 \%$, e os "livros", "sindicatos" e "outros" meios 
correspondem a 13\%. Esses resultados, quando relacionados às investigações de Souza e Paiano (2011), podem identificar que essas dificuldades não são superadas durante o curso de formação e nem mesmo nos estágios obrigatórios, sendo assim, os profissionais acabam se formando com essas dificuldades, superando-as no exercício profissional, sendo que, muitas vezes, isso somente ocorre depois de anos em atividade profissional.

Ao articular as duas dificuldades percebeu-se que os professores são os principais agentes responsáveis por resolveros problemas enfrentados pelos estudantes(ALBUQUERQUE et al., 2009; SILVA, 2011; CÂNDIDO et al., 2014). Esses resultados se coadunam com um estudo recente (TRIANI; NOVIKOFF, 2014) no qual os resultados apontam as dificuldades encontradas pelos estudantes em formação como ameaça e desafio. No entanto, no estudo referenciado, a tendência era de os estudantes agirem de forma passiva e evitarem agir frente à situação problema, mas no estudo em tela os estudantes recorrem aos professores, na maioria dos casos. Portanto, os resultados demonstram que o professor é o meio que os acadêmicos buscam quando há dificuldade político-administrativa ou didático-pedagógica.

\subsection{Valores normativos}

Quando questionados sobre a formação de professores, 44\% dos estudantes não souberam atribuir um sentido à "formação docente" e 13\% responderam de forma "inadequada", isto é, emitiram uma resposta incoerente com a pergunta. Entretanto, $38 \%$ atribuíram sentido à formação docente e ainda $6 \%$ responderam de forma crítica.

Esses dados, referentes ao desconhecimento da formação de professores, também é apontado em outros estudos (TOLENTINO; ROSSO, 2014; CHAMON, 2014). Sendo assim, 0 sentido atribuído sobre o que é ser professor de Educação Física pode implicar representações sociais indesejáveis para a área, como o estudo de Mello e outros (2012), que identificou a Educação Física, na Educação Infantil, representada como um momento de descanso para os professores regentes, e ainda como "disciplina auxiliar" na escola. Portanto, é necessário refletir e engendrar discussões sobre as representações sociais que vêm sendo apresentadas acerca do professor de Educação Física.

Sobre o conhecimento acerca da legislação, apenas $19 \%$ dos estudantes demonstraram conhecer, isso implica dizer que $81 \%$ desconhecem os aspectos legais da formação docente. Em uma segunda análise, percebeu-se que dos estudantes que responderam dominar os conhecimentos legislativos, 33\%, não responderam quais aspectos conhecem, mas 67\% responderam de forma satisfatória, isto é, conhecem alguns artigos e/ou diretrizes concernentes à formação de professores.

Dentre os conhecimentos sobre os aspectos da formação, a fim de investigar a possibilidade de formação continuada, questionou-se o plano dos estudantes para os próximos dois anos e percebeu-se que 50\% ainda não planejaram o que farão após a graduação, 31\% responderam ingressar no "mestrado", 13\% "trocar de curso" e 6\% engajar o "doutorado".

Nessa mesma perspectiva, um estudo recente (LOUREIRO et al., 2015) realizado com professores de Educação Física já formados mostrou que a evocação central sobre formação continuada é atribuída aos elementos "qualificação" e "aprender", condizente com os 37\% dos alunos desta pesquisa que indicaram querer buscar o mestrado e doutorado. Além disso, 
pesquisas apontam que a formação continuada é representada como "troca de experiências" (LEHMKUHL et al., 2007), "crescimento, mudança e participação" (MACHADO, 2008).

O resultado de $50 \%$ não terem um planejamento futuro, isto é, um plano para a formação continuada, também é um fator apontado como problemática em pesquisas recentes (LEHMKUHL et al., 2007; MACHADO, 2008; LOUREIRO et al., 2015).

\subsection{Valores gnosiológicos}

Foi perguntado o que os estudantes acreditam ser importante para a formação do professor e o termo "conhecimento" foi citado por 38\% dos sujeitos, e "formação" por 25\%. Outros fatores emergentes foram "dedicação", "prática" e "dinamismo", com 13\% cada um deles. Além disso, quando questionados sobre as características de um "bom professor" 0 "conhecimento" foi mencionado por $56 \%$ do grupo, o "dinamismo" por $31 \%$ e a "criatividade" por $13 \%$.

Diante dos dados, além das características indicadas pelos graduandos desta pesquisa, um trabalho realizado com estudantes de pedagogia mostrou que a "postura" é representada como característica de um professor (SILVA, 2011). E ainda, em estudo recente (CÂNDIDO et al., 2014), foram identificados resultados semelhantes sobre o "bom professor", no qual a representação social está entre o conhecimento técnico do docente e seu nível de interatividade com o aluno em formação.

Articulando os dados supracitados, percebe-se que para os estudantes o conhecimento é uma característica e fator importante na formação de um bom professor. Esses resultados se coadunam com os de outros autores (PLACCO, 2002; TARDIF; LESSARD, 2005; SOUSA et al., 2009; CUNHA, 2010; TOLENTINO; ROSSO, 2014), pois o termo "conhecimento" corresponde a um forte indício de representação social acerca do ser professor.

Diante das respostas é identificável que para os estudantes a ideia de bom professor está associada ao conhecimento. No entanto, uma representação social sobre o conhecimento associada ao professor pode indicar um racionalismo acadêmico, conforme os resultados de Magalhães Júnior, Lorencini Júnior e Corazza (2013) numa pesquisa com pós-graduandos em ensino de ciências e Negri e seus colaboradores (2015) com licenciandos em Ciências, uma vez que ser um bom professor vai além do conhecimento que ele possui, conforme Placco (2002), Tardif e Lessard (2005) e Sousa e outros (2009) que defendem a interação entre aluno e professor uma das mais importantes características do bom professor.

\subsection{Valores sociorrelacionais}

Ao serem questionados sobre a percepção que têm sobre seus colegas de classe, $50 \%$ dos estudantes "acreditam" na formação a ponto de exercê-la futuramente. Entretanto, $19 \%$ afirmam que os colegas de classe "não acreditam", ou seja, não têm seriedade durante a formação. Outros $25 \%$ disseram não saber qual a percepção dos outros estudantes sobre a formação.

Frente às atividades complementares de maior interesse, o "seminário" foi o mais citado com $35 \%$, em seguida a "palestra" somando $25 \%$, depois em $20 \%$ o "grupo de estudo", na sequência 20\% mencionaram "outras" atividades. Esses dados corroboram os resultados de 
Loureiro e outros (2015), que identificaram a formação continuada como um atributo individual de responsabilidade única e exclusiva do professor, cabendo a ele a escolha das atividades que serão integradas e inseridas à sua formação.

Os conteúdos mais relevantes na formação para os estudantes foram os de "Educação Física Escolar", com 38\% dos sujeitos, depois surge "fitness" com $31 \%$, em seguida $25 \%$ entendem que "esportes" são conhecimentos mais relevantes. Percebe-se ainda que "lutas", "atividade física e envelhecimento" e "psicologia" somam apenas $6 \%$ dos conteúdos. Diante disso, como a coleta de dados foi realizada entre alunos de licenciatura e bacharelado, pode-se perceber que possivelmente alguns dos discentes de licenciatura a representam como "Educação Física Escolar" e os do bacharelado possivelmente o representam como "fitness". Esse resultado corrobora os achados de d'Ávila (2007), que identificou representações sociais sobre licenciatura e bacharelado como profissionais distintos segundo os professores investigados.

Nesse contexto, é importante lembrar que a associação da atividade docente com a prática esportiva corresponde a um paradigma da Educação Física (GHIRALDELLI JÚNIOR, 1989; VARGAS, 1990) que, embora muito se tenha mudado essas representações sociais, ainda existe (VIEIRA, 2006; BARBOSA, 2012; CÂNDIDO et al., 2015; TRIANI et al., 2016).

É importante lembrar que em nenhuma ocasião os temas transversais foram mencionados, algo que pode ser um indício da falta de formação nessa perspectiva, como afirmam Magalhães Júnior e Tomanik (2013) ao realizar investigação com professores acerca do conhecimento sobre meio ambiente.

\subsection{Valores identitários}

foi perguntado aos estudantes o motivo pelo qual ingressaram no curso, e a maior parte deles respondeu que foi por gosto pela área, mencionado por $63 \%$ dos participantes. $16 \%$ apresentaram os valores socioprofissionais. Outros valores não foram significativos.

Neste quesito, destacou-se a afetividade como fator decisivo na escolha do curso de formação de professores em Educação Física, resultado também encontrado em estudo anterior (KRUG; KRUG, 2008). Esses autores mostraram que muitos estudantes de Educação Física escolhem a área de forma inconsciente, além disso, mais da metade dos alunos optam pela área pelo afeto para com as atividades esportivas e físicas. Assim, o gosto pelo esporte ainda constitui um motivo para escolha do curso de Educação Física (RAZEIRA et al., 2014).

Os fatores positivos na vida de estudante foram a "aquisição de conhecimento", com $75 \%$, "formação acadêmica", com 13\%, e "fazer amigos", com 13\%. Nessa mesma perspectiva, os negativos foram "falta de tempo", somando 44\%, "trabalhar e estudar" e "falta de informação", ambas totalizaram 19\%. A "perda de oportunidades" foi citada por 13\%, e 5\% mencionaram que "não há" fatores negativos na vida do estudante.

Em uma autoavaliação, 69\% se classificam como um aluno "bom". Já 19\% classificam-se como "regular". Outros 6\% citam "deficiente" e 6\% foram otimistas em "muito bom".

\section{CONCLUSÃO}

Ao identificar a representação social dos estudantes do curso de Educação Física, no contexto da formação inicial, por meio da Teoria das Representações Sociais, foi perceptível a forma de conhecimento socialmente elaborada e compartilhada, na qual foi encontrado um 
conjunto de valores que, quando articulados, compõem as representações sociais sobre a formação de professores para os estudantes do curso de Educação Física, e essas são de que o professor é o responsável pela resolução dos problemas, sendo que os estudantes, em maioria, não conseguiram atribuir um sentido para formação docente, mesmo sabendo que ter conhecimento é importante para ser um bom profissional. Somente metade dos que estão na graduação acreditam na sua formação, embora tenham ingressado por identificação pela área.

Ao analisar os resultados do questionário nas dimensões valorativas, foi possível identificar que os valores interagem entre si, e essa interação faz emergir um conjunto de representações sociais que são norteadas por valores normativos, gnosiológicos, sociorrelacionais, socioprofissionais e identitários. Sendo assim, essas representações sociais são identificadas de duas formas, a saber: subjetivação e ancoragem (MOSCOVICl, 1978). A primeira surge no momento em que os estudantes expressam seus conhecimentos frente à formação de professores, isto é, até mesmo aqueles de períodos mais avançados não souberam atribuir um sentido à formação de professores, já a segunda torna-se visível quando focalizam a importância do elemento "conhecimento" na formação de professores.

Portanto, sabendo das representações sociais desse grupo específico é cabível questionar se há discussão sobre a formação de professor para esse grupo, uma vez que mais da metade dos participantes não souberam atribuir um sentido à formação de professores. Contudo, caso exista a discussão sobre essa temática, talvez seja oportuno ressignificá-la, a fim de que se alcance uma formação de professores mais crítica e reflexiva e que não tente fazer Educação no silêncio, mas na ação e reflexão do trabalho docente.

\section{REFERÊNCIAS}

ALBUQUERQUE, Igor Valença de et al. Dificuldades encontradas na Educação Física Escolar que influenciam na não-participação dos alunos: reflexões e sugestões. EFDeportes.com Revista Digital, v.14, n.136, set. 2009. Disponível em: < http://www.efdeportes.com/efd136/dificuldades-encontradasna-educacao-fisica-escolar.htm>. Acesso em: 20 mar. 2016.

BARDIN, Laurence. Análise de Conteúdo. Lisboa: Edições 70, 2009.

BAUER, Martin; GASKELL, George. Pesquisa qualitativa com texto, imagem e som: um manual prático. 2.ed. Petrópolis: Vozes, 2003.

CÂNDIDO, Cássia Marques et al. A representação social do "bom professor" no ensino superior. Psicologia \& Sociedade, v.26, n.2, p.356-365, 2014.

CÂNDIDO, Cássia Marques et al. Representação da Educação Física na 18ª temporada da telenovela Malhação. Revista Brasileira de Educação Física e Esporte, v.29, n.1, p.95-106, 2015.

CHAMON, Edna Maria Querido de Oliveira. Representações sociais da formação docente em estudantes e professores da Educação Básica. Psicologia Escolar e Educacional, v.18, n.2, p.303312, 2014.

CRESWELL, John. Projeto de Pesquisa: métodos qualitativo, quantitativo e misto. Porto Alegre: 
CUNHA, Antônio Camilo. Representação do "bom" professor: o "bom" professor em geral e o "bom" professor de educação física em particular. Educação em Revista, v.11, n.2, p.41-52, jul. /dez. 2010.

CZAJA, Ronald; JOHNNY, Blair. Designing Surveys: a Guide to Decisions and Procedures. Thousand Oaks: Pine Forge , 1996.

DURKHEIM, Émile. As regras do método sociológico. 13. ed. São Paulo: Editora Nacional, 1987.

D’ÁVILA, Ronaldo Castro. As representações sociais dos professores de graduação em educação física sobre o bacharelado e a licenciatura. 2007. 167f. Dissertação (Mestrado). Belo Horizonte: Faculdade de Educação da UFMG, 2007.

GHIRALDELLI JÚNIOR, Paulo. Educação Física progressista: a pedagogia crítico-social dos conteúdos e a Educação Física brasileira. São Paulo: Loyola, 1989.

GIBBONS, Michael et al. The new production of knowledge: the dynamics of science and research in contemporary societies. London: Sage, 1994.

GÜNTHER, Hartmut. Pesquisa qualitativa versus pesquisa quantitativa: esta é a questão? Psicologia: Teoria e Pesquisa, v. 22, n. 2, p. 201-210, 2006.

HOROCHOVSKI, Marisete Teresinha Hoffmann. Representações Sociais: Delineamentos de uma Categoria Analítica. Revista Eletrônica dos Pós-Graduandos em Sociologia Política da UFSC, v.2, n 1-2, p.92-106, jan. /jun. 2004.

JODELET, Denise (Org.). As representações sociais. Rio de Janeiro: Ed. Uerj, 2001.

KRUG, Rodrigo de Rosso; KRUG, Hugo Norberto. Os diferentes motivos da escolha da Licenciatura em Educação Física pelos acadêmicos do CEFD/UFSM. EFDeportes.com, Revista Digital v. 13, n. 123, ago. 2008. Disponível em: < http://www.efdeportes.com/efd123/os-diferentes-motivos-da-escolhada-licenciatura-em-educacao-fisica.htm >. Acesso em: 16 fev. 2016.

LEHMKUHL, Isolene; RAFAELI, Kátia Solange Coelho; SILVA, Neide de Melo Aguiar. Representações sociais dos professores das séries iniciais: saberes sobre a própria formação. In: CONGRESSO NACIONAL DE EDUCACAM, 7., 2007. Anais ... Curitiba: PUC-PR, 2007. p. 200.

LOUREIRO, Walk; CAPARROZ, Francisco Eduardo; BRACHT, Valter. A representação social de formação continuada de professores de Educação Física da rede estadual do Espírito Santo. Revista Brasileira de Educação Física e Esporte, v.29, n.4, p.571-581, 2015.

MACHADO, Laêda Bezerra. Representações sociais, educação e formação docente: tendências e pesquisas na IV Jornada Internacional. Educação por extenso em Foco, 2008. Disponível em: < http://www.fundaj.gov.br/geral/educacao foco/representantessociaislaedamachado.pdf>. Acesso em: 20 mar. 2016.

MAGALHÃES JÚNIOR, Carlos Alberto de Oliveira; LORENCINI JÚNIOR, Alvaro; CORAZZA, Maria Júlia. Representações sociais de pós-graduandos sobre "ser professor de ciências". Enseñanza de las Ciencias, v.31, n.1, p. 2070-2074, 2013.

MAGALHÃES JÚNIOR, Carlos Alberto de Oliveira; TOMANIK, Eduardo Augusto. Representações sociais de meio ambiente: subsídios para a formação continuada de professores. Ciência \&\& Educação, v.19, n.1, p.181-199, 2013.

MAZZOTTI, Tarso Bonilha. Representação social de "problema ambiental": uma contribuiç̧ão à educação ambiental. Revista Brasileira de Estudos Pedagógicos, v.78, n.188/190, p. 86-123, jan./dez. 1997. 
MELLO, André Silva et al. Representações sociais sobre a educação física na educação infantil. Revista da Educação Física/ UEM , v.23, n.3, p.443-455, jul./set. 2012.

MOSCOVICl, Serge. A Representação Social sobre a Psicanálise. Rio de Janeiro: Zahar, 1978.

MOSCOVICI, Serge. A Psicanálise, sua imagem e seu público. Petrópolis, RJ: Vozes, 2012.

NEGRI, Heloisa Fernanda Ortencio et al. Representação Social e Formação de Professores de Ciências. UNOPAR Científica: Ciências Humanas e Educação, v. 1, n. 1, p. 29-33, 2015.

NOVIKOFF, Cristina. Dimensões Novikoff: um constructo para o ensino-aprendizado da pesquisa. In: ROCHA, José Geraldo da; NOVIKOFF, Cristina (Orgs.). Desafios da práxis educacional à promoção humana na contemporaneidade. Rio de Janeiro: Espalhafato Comunicação, 2010. p. 211-242.

NOVIKOFF, Cristina. As representações sociais acerca de ensino superior para professores de graduação da área da saúde. 2006. 205f. Tese (Doutorado). - São Paulo: Pontifícia Universidade Católica de São Paulo, 2006.

OLIVEIRA, Márcio. Representação social e simbolismo: os novos rumos da imaginação na sociologia brasileira. Revista de ciências humanas, n.7/8, p.173-193, 1999.

PLACCO, Vera Maria de Souza. Formação e prática do educador e do orientador. Campinas, SP: Papirus, 2002.

RAZEIRA, Maurício Berndt et al. Os motivos que levam à escolha do curso de licenciatura em educação física e as pretensas áreas de atuação. Revista Mackenzie de Educação Física e Esporte, v. 13, n. 2, p. 124-136, jul./dez. 2014.

SILVA, Ariane Franco Lopes da. Corporeidade e representações sociais: agir e pensar a docência. Psicologia \& Sociedade por extenso, v.23, n.3, p.616-624, 2011.

SILVA, Verônica Santos; SANTOS, Cristiane; DIAS, Alfrancio Ferreira. Relato de experiência: os principais desafios e dilemas vivenciados pelos discentes do curso de pedagogia durante o estágio supervisionado. In: SEMANA DE PEDAGOGIA DA UNIVERSIDADE ESTADUAL DO SUDESTE DA BAHIA, 1., 2012. Anais... Jequié: Universidade Estadual do Sudeste da Bahia, 2012. p.1-11.

SOUSA, Clarilza Prado de; LIMA, Laura Camara; SILVA, Ariane Franco Lopes da. Educación y alteridade: la relación profesor-alumno vista por los Estudiantes de pedagogía. Revista Investigaciones en Psicología, v. 14, n.2, p. 151-171, 2009.

SOUZA, Flávia Aparecida de; PAIANO, Marcelle. Desafios e dificuldades enfrentadas pelos profissionais de Enfermagem em início de carreira. Revista Mineira de Enfermagem, v.15, n.2, p. 267-273, 2011.

TARDIF, Maurice; LESSARD, Claude. 0 trabalho docente: elementos para uma teoria da docência como profissão de interações humanas. Petrópolis, RJ: Vozes, 2005.

TOLENTINO, Patrícia Caldeira; ROSSO, Ademir José. As representações sociais dos licenciandos em ciências biológicas sobre o ser biólogo e o ser professor. Ensaio Pesquisa em Educação em Ciências, v.16, n.3, p.15-34, 2014.

TRIANI, Felipe da Silva; FREITAS, Willian Costa de; NOVIKOFF, Cristina. Educação Física: as representações sociais. Conexões: revista da Faculdade de Educação Física da UNICAMP, v. 14, n. 1, p. 130-137, jan./mar. 2016. 
TRIANI, Felipe da Silva; NOVIKOFF, Cristina. As formas de valoração e afrontamento na formação de professores (inicial) dos estudantes dos cursos de educação física. Revista práxis, v. 6, n. 11, p.9510, jun. 2014.

TRIANI, Felipe da Silva; NOVIKOFF, Cristina. O estado do conhecimento face às representações sociais sobre formação de professores (inicial) de Educação Física. Revista Carioca de Educação Física, n. 8, p.1-14, 2013.

VARGAS, Angelo Luis de Souza. A Educação Física e o Corpo: a busca da identidade. Rio de Janeiro: Sprint, 1990.

VIEIRA, Flavia Gonzaga Lopes. As representações sociais da educação física escolar. Revista Brasileira de Educação Física e Esporte, v.20, p.349-376, set. 2006.

WAGNER, Wolfgang. Sócio-Gênese e Características das Representações Sociais. In: MOREIRA, Antonia Silva Paredes; OLIVEIRA, Denize Cristina de (Orgs.). Estudos Interdisciplinares de

Representação Social. 2. ed. Goiânia: AB, 1998. p.3-25. 
